\title{
Distributed Control of Mobility \& Routing in Networks of Robots
}

\author{
Michael M. Zavlanos, Alejandro Ribeiro, and George J. Pappas
}

\begin{abstract}
Most coordinated tasks performed by teams of mobile robots, require reliable communications between the members of the team. Therefore, task accomplishment requires that robots navigate their environment with their collective movement restricted to formations that guarantee integrity of the communication network. Maintaining this communication capability induces physical constraints on trajectories but also requires determination of communication variables like routes and transmitted powers. In this paper, we address this problem using a distributed hybrid approach, where continuous motion controllers based on potential fields interact with discrete distributed optimization of the communication variables to result in a muti-robot network that ensures integrity of communications. Our definition of network integrity is defined as the ability of a network to support desired communication rates.
\end{abstract}

\section{INTRODUCTION}

Mobile robot networks have recently emerged as an inexpensive and robust way to address a wide variety of tasks ranging from exploration, surveillance and reconnaissance, to cooperative construction and manipulation. Efficient information exchange and coordination between members of the team are critical for successful completion of these tasks. E.g., recent work on distributed consensus and state agreement has strongly depended on multi-hop communication for convergence and performance guarantees [1].

Multi-hop communication in multi-robot systems has typically relied on constructs from graph theory, with proximity graphs gaining the most popularity. This is consistent with early approaches to wireless networking that used disk models to abstract the physical layer [2]. In this context, communication becomes equivalent to topological connectivity, defined as the property of a graph to transmit information between all pairs of its nodes. Preservation and control of topological connectivity has recently gained increased attention with approaches that strictly maintain communication links $[3,4]$ being followed by least restrictive ones that allow links to be lost [5]. In terms of solution techniques, these are centralized [4] or distributed [3,5] and have been successfully applied to multi-robot control coordination [3].

Although graphs provide a simple abstraction of interrobot communications, it has long being recognized that since links in a wireless network do not entail tangible connections, associating links with arcs on a graph can

The work of Michael M. Zavlanos is supported by the NSF CNS Grant \#1054604. The work of George J. Pappas and Alejandro Ribeiro is supported by the ONR HUNT MURI project.

Michael M. Zavlanos is with the Dept. of Mechanical Engineering, Stevens Institute of Technology, Hoboken, NJ 07030, USA michael.zavlanosestevens.edu. Alejandro Ribeiro and George J. Pappas are with the Dept. of Electrical and Systems Engineering, University of Pennsylvania, Philadelphia, PA 19104, USA \{aribeiro, pappasg\}@seas.upenn.edu. be somewhat arbitrary. Indeed, topological definitions of connectivity start by setting target signal strengths to draw the corresponding graph. Even small signal variations might result in dramatic differences in network topology [6].

In this paper, we employ a simple, yet effective, modification that relies on weighted graph models with weights that capture the packet error probability of each link [7]. When using reliabilities as link metrics it is possible to model routing and scheduling problems as optimization problems that accept link reliabilities as inputs [8]. The key idea proposed in this paper is to define connectivity in terms of communication rates and to use optimization formulations to describe optimal operating points of wireless networks [8]. In particular, we decompose control in the communication and physical domains, so that the communication variables are updated in discrete time based on distributed dual decomposition techniques, while robot motion is regulated in continuous time by means of appropriate barrier potentials that maintain desired communication rates. Composition of the above, results in a distributed multi-robot hybrid system for which we show that desired communication rates are always guaranteed. The challenge we need to address is that dual decomposition ensures feasibility of the primal variables only asymptotically. This precludes verbatim use of those variables in barrier potentials in the physical domain and gives rise to an infeasibility gap, which results in approximate communication guarantees.

\section{Optimal Wireless COMMUNICATIONS}

Consider a mobile network composed of $J$ robots and a fixed infrastructure with $K$ access points (APs). The robots move throughout an area of interest to accomplish an assigned task for which it is necessary to maintain reliable communications with the infrastructure. Due to, e.g., power constraints or an adverse propagation environment, robots collaborate to maintain a multihop network with the APs. Denote as $\mathbf{x}_{j}$ for $j=1, \ldots, J$ the position of the robots and $\mathbf{x}_{j}$ for $j=J+1, \ldots, J+K$ the position of the APs. The set of all positions $\mathbf{x}:=\left\{\mathbf{x}_{i}\right\}_{i=1}^{J+K}$ is referred to as the spatial configuration of the network. We model communication by a link reliability metric $R(\mathbf{x}, \mathbf{y})$ denoting the probability that a packet transmitted from a terminal located at position $\mathbf{x}$ is correctly decoded by a terminal at position $\mathbf{y}$. This function determines the probability $R_{i j} \triangleq R\left(\mathbf{x}_{i}, \mathbf{x}_{j}\right)$ with which a packet transmitted by node $i$ is correctly decoded by node $j$. Node $j$ is a robot if $j \leq J$ or an AP otherwise.

We further denote as $r_{i}$ the average rate at which robot $i$ delivers information to the APs. If robot $i$ can reach some of the APs, packets are directly conveyed to the 


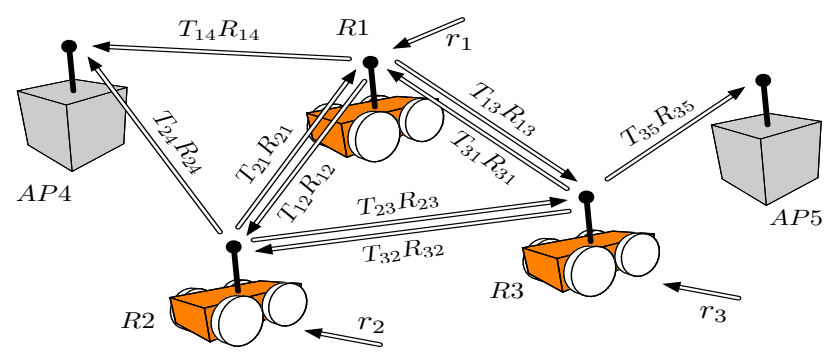

Fig. 1. Robotic network consisting of two access points (AP) and three robots $(\mathrm{R})$. Shown are the packet rates $r_{i}$ generated by every robot as well as the rates $T_{i j} R\left(\mathbf{x}_{i}, \mathbf{x}_{j}\right)$ sent from robot $i$ and successfully decoded by robot $j$, where $T_{i j}$ is the probability that robot $i$ routes packets to robot $j$ and $R\left(\mathbf{x}_{i}, \mathbf{x}_{j}\right)$ is the reliability of the channel between robots $i$ and $j$.

corresponding AP. Otherwise, packets are routed to another robot for subsequent transmission. We model this process through the introduction of routing probabilities $T_{i j}$ denoting the probability with which robot $i$ selects node $j$, a robot or an AP, as a destination of its transmitted packets; see Fig. 1.

Between the time of their generation or arrival from another robot and their transmission packets are stored in a queue. To ensure stability of these queues it suffices to require the average rate at which packets arrive at the $i$ th queue to be smaller than the average rate at which packets leave this queue. Thus, our interest is to determine routing probabilities $T_{i j}$ and rates $r_{i}$ that satisfy the inequality

$$
r_{i}+\sum_{j=1}^{J} T_{j i} R\left(\mathbf{x}_{j}, \mathbf{x}_{i}\right) \leq \sum_{j=1}^{J+K} T_{i j} R\left(\mathbf{x}_{i}, \mathbf{x}_{j}\right) .
$$

Any set of variables $\left\{r_{i}\right\}_{\forall i}$ and $\left\{T_{i j}\right\}_{\forall i, j}$ that satisfy the inequalities in (1) ensures information delivery. A basic requirement is that all robots can communicate with the infrastructure APs at least at a basal rate of $r_{i 0}$ packets per time unit. When this happens we say that we have network integrity as we formally define next.

Definition 1 (Network integrity) For configuration $\mathbf{x}$ and basal rates $r_{i 0}$, network integrity implies not only existence but also availability of rates $\left\{r_{i}\right\}_{\forall i}$ and routing variables $\left\{T_{i j}\right\}_{\forall i, j}$ for which the inequalities in (1) hold and $r_{i} \geq r_{i 0}$.

For a given spatial configuration $\left\{\mathbf{x}_{i}\right\}_{i=1}^{J+K}$ there might be various sets of variables that ensure network integrity. To select an element of this set we introduce strictly concave optimality criteria $U_{i}\left(r_{i}\right)$ and $V_{i j}\left(T_{i j}\right)$ measuring the value associated with variables $r_{i}$ and $T_{i j}$ respectively. The operating point is then selected as the solution of the optimization problem

$$
\begin{aligned}
P_{\mathbf{x}}= & \max _{T_{i j}} \sum_{i=1}^{J} U_{i}\left(r_{i}\right)+\sum_{i=1}^{J} \sum_{j=1}^{J+K} V_{i j}\left(T_{i j}\right) \\
\text { s.t } \quad & r_{i}+\sum_{j=1}^{J} T_{j i} R\left(\mathbf{x}_{j}, \mathbf{x}_{i}\right) \leq \sum_{j=1}^{J+K} T_{i j} R\left(\mathbf{x}_{i}, \mathbf{x}_{j}\right) \\
& r_{i} \geq r_{i 0}, \quad \sum_{j=1}^{J} T_{i j} \leq 1
\end{aligned}
$$

where the constraints are required for all $i \in\{1, \ldots, K\}$. To ensure network integrity for configuration $\mathbf{x}$, we need to find optimal routing probabilities $T_{i j}$ that solve the optimization problem in (2). This yields basal rates $r_{i 0}$ for all terminals, while assigning the remaining resources in a manner that is optimal in terms of utilities $U_{i}\left(r_{i}\right)$ and $V_{i j}\left(T_{i j}\right)$.

\section{A. Distributed Optimal Communication}

Solving (2) at a central designated node entails a large communication cost to convey the network's topology and disseminate the optimal operating point. This cost can be avoided by devising a distributed solution based on the separability of the Lagrangian dual of (2). To do so consider the optimal communication problem in (2), associate Lagrange multipliers $\lambda_{i}$ with each of the routing constraints in (1), and define the Lagrangian as

$$
\begin{aligned}
& \mathcal{L}_{\mathbf{x}}(\boldsymbol{\lambda}, \mathbf{T}, \mathbf{r})=\sum_{i=1}^{J} U_{i}\left(r_{i}\right)+\sum_{i=1}^{J} \sum_{j=1}^{J+K} V_{i j}\left(T_{i j}\right) \\
& \quad+\lambda_{i}\left[\sum_{j=1}^{J+K} T_{i j} R\left(\mathbf{x}_{i}, \mathbf{x}_{j}\right)-T_{j i} R\left(\mathbf{x}_{j}, \mathbf{x}_{i}\right)-r_{i}\right] .
\end{aligned}
$$

The dual function is then defined as the maximum of the Lagrangian with respect to primal variables, i.e.,

$$
g_{\mathbf{x}}(\boldsymbol{\lambda})=\operatorname{argmax}_{r_{i} \geq r_{i 0}, \sum_{j=1}^{J} T_{i j} \leq 1} \mathcal{L}_{\mathbf{x}}(\boldsymbol{\lambda}, \mathbf{T}, \mathbf{r}) .
$$

The dual problem is finally defined as the minimization of the dual function, $D_{\mathbf{x}}=\min _{\boldsymbol{\lambda} \geq \mathbf{0}} g_{\mathbf{x}}(\boldsymbol{\lambda})$. Since for fixed spatial configurations $\mathbf{x}$, the problem in (2) is convex it holds that $D_{\mathbf{x}}=P_{\mathbf{x}}$ implying that we can work with the dual problem in lieu of the primal problem in (2). In particular, a distributed algorithm can be obtained by implementing gradient descent in the dual domain.

To implement dual gradient descent we compute the gradient of the dual function using primal Lagrangian maximizers, see e.g., [9]. For given $\boldsymbol{\lambda}$ define the primal Lagrangian maximizers as

$$
\left\{r_{i}(\boldsymbol{\lambda})\right\}_{\forall i},\left\{T_{i j}(\boldsymbol{\lambda})\right\}_{\forall i, j}:=\underset{\substack{r_{i} \geq r_{i 0}, \sum_{j=1}^{J} T_{i j} \leq 1}}{\operatorname{argmax}} \mathcal{L}_{\mathbf{x}}(\boldsymbol{\lambda}, \mathbf{T}, \mathbf{r}) .
$$

The components of the dual function's gradient are then given by the constraint slack associated with $\left\{r_{i}(\boldsymbol{\lambda})\right\}_{\forall i}$ and $\left\{T_{i j}(\boldsymbol{\lambda})\right\}_{\forall i, j}$, i.e.,

$$
\left[\nabla g_{\mathbf{x}}(\boldsymbol{\lambda})\right]_{i}=\sum_{j=1}^{J+K} T_{i j}(\boldsymbol{\lambda}) R\left(\mathbf{x}_{i}, \mathbf{x}_{j}\right)-T_{j i}(\boldsymbol{\lambda}) R\left(\mathbf{x}_{j}, \mathbf{x}_{i}\right)-r_{i}(\boldsymbol{\lambda}) .
$$

A key observation here is that the Lagrangian in (3) can be written as a sum of local Lagrangians that depend only on variables $r_{i}$ and $\left\{T_{i j}\right\}_{\forall i}$. Indeed, it suffices to reorder terms in (3) to realize that upon defining local Lagrangians

$$
\mathcal{L}_{i}(\boldsymbol{\lambda}, \mathbf{T}, \mathbf{r})=U_{i}\left(r_{i}\right)-\lambda_{i} r_{i}+\sum_{j=1}^{J+K} T_{i j} R\left(\mathbf{x}_{i}, \mathbf{x}_{j}\right)\left(\lambda_{i}-\lambda_{j}\right),
$$

it is possible to write

$$
\mathcal{L}(\boldsymbol{\lambda}, \mathbf{T}, \mathbf{r})=\sum_{i=1}^{J} \mathcal{L}_{i}(\boldsymbol{\lambda}, \mathbf{T}, \mathbf{r})
$$

The local Lagrangian $\mathcal{L}_{i}(\boldsymbol{\lambda}, \mathbf{T}, \mathbf{r})$ is defined so that all summands of the global Lagrangian $\mathcal{L}(\boldsymbol{\lambda}, \mathbf{T}, \mathbf{r})$ that involve 
primal variables $r_{i}$ and $\left\{T_{i j}\right\}_{j=1}^{J+K}$ for given $i$ appear in, and only in, $\mathcal{L}_{i}(\boldsymbol{\lambda}, \mathbf{T}, \mathbf{r})$ [cf. (3) and (7)]. Therefore, to find the variables $r_{i}(\boldsymbol{\lambda})$ and $\left\{T_{i j}(\boldsymbol{\lambda})\right\}_{j=1}^{J+K}$ that maximize the global Lagrangian as per (5) it suffices to find the arguments that maximize the local Lagrangian in (7).

Introduce now an index $n$ and consider times $t_{n}$ at which variables are updated. We can use the observation in (9) to write the following distributed gradient descent algorithm for the dual function:

Primal iteration For given Lagrange multipliers $\boldsymbol{\lambda}\left(t_{n}\right)$ compute Lagrangian maximizers $r_{i}\left(t_{n}\right)$ and $\left\{T_{i j}\left(t_{n}\right)\right\}_{j=1}^{J+K}$ as

$$
r_{i}\left(t_{n}\right),\left\{T_{i j}\left(t_{n}\right)\right\}_{j=1}^{J+K}=\underset{\substack{r_{i} \geq r_{i 0}, \sum_{j=1}^{J} T_{i j} \leq 1}}{\operatorname{argmax}} \mathcal{L}_{i}\left(\boldsymbol{\lambda}\left(t_{n}\right), \mathbf{T}, \mathbf{r}\right) .
$$

Dual Iteration. Use the primal variables $r_{i}\left(t_{n}\right)$ and $\left\{T_{i j}\left(t_{n}\right)\right\}_{j=1}^{J+K}$ in (9) to update the dual variables as

$$
\begin{aligned}
& \lambda_{i}\left(t_{n+1}\right)=P\left[\lambda_{i}\left(t_{n}\right)-\right. \\
& \left.\quad \epsilon\left[\sum_{j=1}^{J+K} T_{i j}\left(t_{n}\right) R\left(\mathbf{x}_{i}, \mathbf{x}_{j}\right)-T_{j i}\left(t_{n}\right) R\left(\mathbf{x}_{j}, \mathbf{x}_{i}\right)-r_{i}\left(t_{n}\right)\right]\right]
\end{aligned}
$$

where the operator $P[\cdot]$ denotes projection on the nonnegative reals.

Letting variables $r_{i}\left(t_{n}\right),\left\{T_{i j}\left(t_{n}\right)\right\}_{j=1}^{J+K}$, and $\lambda_{i}\left(t_{n}\right)$ be associated with terminal $i$, algorithm (9)-(10) can be implemented in a distributed manner. The maximization in (9) requires access to local multipliers $\lambda_{i}$ and multipliers $\lambda_{j}$ from those terminals for which $R\left(\mathbf{x}_{i}, \mathbf{x}_{j}\right) \neq 0$. Since these terminals can communicate with $i$, these multipliers can be conveyed to let $i$ compute its primal variables of interest. Likewise, the dual update in (10) requires access to local primal variables $\left\{T_{i j}\left(t_{n}\right)\right\}_{j=1}^{J+K}$ and primal variables from terminals that can communicate directly with $i$.

\section{Distributed Motion \& COMmunicAtion CONTROL}

In Section II, we showed that for fixed robot position $\mathbf{x}_{i}$, the reliabilities $R\left(\mathbf{x}_{i}, \mathbf{x}_{j}\right)$ are fixed and the problem in (2) attains a simple convex form. However, this is not the case for mobile robots that are supposed to move to accomplish their assigned task. In particular, we consider single integrator robots whose positions $\mathbf{x}_{i}(t)$ react to control inputs $\mathbf{u}_{i}(t)$ according to the first order differential equations

$$
\dot{\mathbf{x}}_{i}(t)=\mathbf{u}_{i}\left(t ; \sigma_{i}\left(t_{n}\right)\right), \quad \forall t \in\left[t_{n}, t_{n+1}\right),
$$

for all $i=1, \ldots, J$, where

$$
\sigma_{i}(t)=\left\{r_{i}(t),\left\{T_{i j}(t)\right\}_{j=1}^{J+K}\right\} \cup\left\{T_{j i}(t)\right\}_{j=1}^{J}
$$

denotes the collection of communication variables that are either locally computed at robot $i$ by the primal-dual iteration (9)-(10), i.e., the rates $r_{i}(t)$ and routes $\left\{T_{i j}(t)\right\}_{j=1}^{J+K}$, or are received by robot $i$ 's neighbors via communication, i.e., the routes $\left\{T_{j i}(t)\right\}_{j=1}^{J}$. In (11), $\left\{t_{n}\right\}_{n=0}^{\infty}$ denotes the sequence of time instants when these updates and receptions take place. In the above formulation, the communication variables and Lagrange multipliers are piecewise constant functions of time, so that $T_{i j}(t)=T_{i j}\left(t_{n}\right), r_{i}(t)=r_{i}\left(t_{n}\right)$ and $\lambda_{i}(t)=\lambda_{i}\left(t_{n}\right)$ for all $t \in\left[t_{n}, t_{n+1}\right)$ and all indices $i$ and $j$, and so is the switching signal $\sigma_{i}(t)$. To the contrary, the channel reliabilities $R\left(\mathbf{x}_{i}(t), \mathbf{x}_{j}(t)\right)$ are continuous functions of time that depend on the robot positions $\mathbf{x}(t)$ in (11).

To design motion controllers $\mathbf{u}_{i}\left(t ; \sigma_{i}\left(t_{n}\right)\right)$ for the robots, we classify them in "leaders" and "relays" so that leaders are assigned a task that they need to complete, while relays only relay the information that the leaders generate in the network. Let $\hat{\phi}_{i}: \mathbb{R}^{J d} \rightarrow \mathbb{R}_{+}$be an artificial potential function with

$$
\hat{\phi}_{i}\left(t ; \sigma_{i}\left(t_{n}\right)\right) \triangleq\left\{\begin{array}{ll}
\frac{\gamma_{i}(t)}{\beta_{i}\left(t ; \sigma_{i}\left(t_{n}\right)\right)}, & \text { if } i \text { is a leader robot } \\
\frac{1}{\beta_{i}\left(t ; \sigma_{i}\left(t_{n}\right)\right)}, & \text { if } i \text { is a relay robot }
\end{array},\right.
$$

where $\gamma_{i}: \mathbb{R}^{d} \rightarrow \mathbb{R}_{+}$is a task potential that drives, for example, a leader robot to its target and $\beta_{i}: \mathbb{R}^{J d} \rightarrow \mathbb{R}_{+}$ with

$$
\begin{aligned}
\beta_{i}\left(t ; \sigma_{i}\left(t_{n}\right)\right) \triangleq & \sum_{j=1}^{J+K} T_{i j}\left(t_{n}\right) R\left(\mathbf{x}_{i}(t), \mathbf{x}_{j}(t)\right) \\
& -\sum_{j=1}^{J} T_{j i}\left(t_{n}\right) R\left(\mathbf{x}_{j}(t), \mathbf{x}_{i}(t)\right)-r_{i}\left(t_{n}\right)
\end{aligned}
$$

is a potential that measures satisfaction of the queue balance constraint associated with the queue at robot $i$. Since $\hat{\phi}_{i}$ can grow unbounded as the queue balance constraints tend to become violated, i.e., as $\beta_{i} \rightarrow 0$, we further introduce a diffeomorphism $\psi:[0, \infty] \rightarrow[0,1]$ with $\psi(y)=y /(1+y)$ that "squashes" the image of $\hat{\phi}_{i}$ from $[0, \infty]$ to $[0,1]$. Let $\phi_{i}: \mathbb{R}^{J d} \rightarrow[0,1]$ denote the resulting potential such that

$$
\phi_{i}=\psi \circ \hat{\phi}_{i}=\left\{\begin{array}{ll}
\frac{\gamma_{i}}{\gamma_{i}+\beta_{i}}, & \text { if } i \text { is a leader robot } \\
\frac{1}{1+\beta_{i}}, & \text { if } i \text { is a relay robot }
\end{array},\right.
$$

and for every robot $i$, whether a leader or a relay, let

$$
\mathbf{u}_{i}\left(t ; \sigma_{i}\left(t_{n}\right)\right)=-k \nabla_{\mathbf{x}_{i}} \phi_{i}\left(t ; \sigma_{i}\left(t_{n}\right)\right),
$$

for all $t \in\left[t_{n}, t_{n+1}\right)$, denote the control law, where $k>0$ is a positive gain to regulate the robot speed.

\section{A. Distributed Integration}

The proposed distributed primal-dual iteration (9)-(10) to solve problem (2) only ensures feasibility of the primal variables in the limit, which presents practical difficulties in ensuring that $\beta_{i}>0$ in (12) without bringing the system (robots) to an almost complete halt. For this, we propose an integration scheme where the queue balance constraints (1) are satisfied approximately, with some bounded error.

Problem 1 Determine a sequence of communication variables $\left\{\sigma_{i}\left(t_{n}\right)\right\}_{n=0}^{\infty}$ and a set of distributed motion controllers $\mathbf{u}_{i}\left(t ; \sigma_{i}\left(t_{n}\right)\right)$ for all robots $i$ so that communication rates $r_{i}(t)$ approximately exceed $r_{i 0}$ along the trajectories of the closed loop system (11), at all times.

In other words, Problem 1 implies that $r_{i}(t) \geq r_{i 0}-e_{i}\left(t_{n}\right)$ for all time $t \geq 0$ and for a small positive error $e_{i}\left(t_{n}\right)>$ 0 that, in general, may depend on the sequence $\left\{t_{n}\right\}_{n=0}^{\infty}$. 
The error $e_{i}\left(t_{n}\right)>0$ essentially captures the feasibility gap in the queue balance constraints (1) after a single primaldual iteration (9)-(10) at time $t_{n}$. This feasibility gap is also introduced in the motion controllers $\mathbf{u}_{i}\left(t ; \sigma_{i}\left(t_{n}\right)\right)$ defined in (14) via the barrier potential functions

$$
\tilde{\beta}_{i}\left(t ; \sigma_{i}\left(t_{n}\right)\right) \triangleq \beta_{i}\left(t ; \sigma_{i}\left(t_{n}\right)\right)+e_{i}\left(t_{n}\right),
$$

where $\beta_{i}\left(t ; \sigma_{i}\left(t_{n}\right)\right)$ is defined as in (12). Integration of the closed loop system (11)-(14) for motion control with the primal-dual iterations (9)-(10) for optimal communication leads to a distributed motion and communication control algorithm. The algorithm consists of the primal-dual iteration (9)-(10) that updates the communication variables, and an update in the robot positions via the closed loop system (11)(14). At times $\left\{t_{n}\right\}_{n=0}^{\infty}$ communication between neighbors takes place to provide robots with the variables that are necessary in their updates.

\section{Algorithm PerformanCE}

To simplify presentation introduce a vector $\mathbf{t}$ stacking the rows of the transmission probability matrix $\mathbf{T}$ and a matrix $\mathbf{A}_{\mathbf{x}}$ with dimensions $J \times J(J+K)$ so as to write the constraints in (1) as $\mathbf{A}_{\mathbf{x}} \mathbf{t}-\mathbf{r} \geq \mathbf{0}$. Using this definition we can rewrite (2) as

$$
P_{\mathbf{x}}=\max f_{0}(\mathbf{r}, \mathbf{t}), \quad \text { s.t. } \mathbf{A}_{\mathbf{x}} \mathbf{t}-\mathbf{r} \geq \mathbf{0},
$$

where constraints $r_{i} \geq r_{i 0}$ and $\sum_{j=1}^{J} T_{i j} \leq 1$ are implicit. Similarly, we using this notation we can rewrite the optimal distributed communication algorithm in (9) and (10) as

$$
\begin{aligned}
& \mathbf{t}\left(t_{n}\right), \mathbf{r}\left(t_{n}\right)=\operatorname{argmax} \mathcal{L}_{i}\left(\boldsymbol{\lambda}\left(t_{n}\right), \mathbf{t}, \mathbf{r}\right), \\
& \boldsymbol{\lambda}\left(t_{n+1}\right)=P\left[\boldsymbol{\lambda}\left(t_{n}\right)-\epsilon\left(\mathbf{A}_{\mathbf{x}\left(t_{n}\right)} \mathbf{t}\left(t_{n}\right)-\mathbf{r}\left(t_{n}\right)\right)\right] .
\end{aligned}
$$

In a static setting, i.e., for fixed robot positions, dual variables $\boldsymbol{\lambda}\left(t_{n}\right)$ approach the optimal multipliers $\boldsymbol{\lambda}_{\mathbf{x}\left(t_{n}\right)}^{*}$. Provided some technical conditions, convergence of the primal variables $\mathbf{t}\left(t_{n}\right)$ and $\mathbf{r}\left(t_{n}\right)$ to the optimal network operating point $\mathbf{t}_{\mathbf{x}\left(t_{n}\right)}^{*}, \mathbf{r}_{\mathbf{x}\left(t_{n}\right)}^{*}$ follows as a consequence [9]. In the dynamic setting considered here, the primal and dual variable updates in (17) bring the network closer to its optimal operating point. However when terminals move as per (11)-(14), the optimal operating point drifts away towards $\mathbf{t}_{\mathbf{x}\left(t_{n+1}\right)}^{*}, \mathbf{r}_{\mathbf{x}\left(t_{n+1}\right)}^{*}$. Our goal is to determine the optimality of the operating point $\mathbf{t}\left(t_{n}\right), \mathbf{r}\left(t_{n}\right)$ with respect to the optimal operating point $\mathbf{t}_{\mathbf{x}\left(t_{n}\right)}^{*}, \mathbf{r}_{\mathbf{x}\left(t_{n}\right)}^{*}$ for the current team configuration $\mathbf{x}\left(t_{n}\right)$.

Characterizing operating point $\mathbf{t}\left(t_{n}\right), \mathbf{r}\left(t_{n}\right)$ concerns determination of feasibility and optimality. Throughout the subsequent analysis we make the following assumptions on the dual functions $g_{\mathbf{x}}(\boldsymbol{\lambda})$ :

(A1) The dual functions $g_{\mathbf{x}}(\boldsymbol{\lambda})$ are strongly convex for all $\mathbf{x}$ with strong convexity parameter $m$,

$$
g_{\mathbf{x}}(\boldsymbol{\mu}) \geq g_{\mathbf{x}}(\boldsymbol{\lambda})+\nabla g_{\mathbf{x}}(\boldsymbol{\lambda})^{T}(\boldsymbol{\mu}-\boldsymbol{\lambda})+\frac{m}{2}\|\boldsymbol{\lambda}-\boldsymbol{\mu}\|^{2}
$$

(A2) The gradients of the dual functions $g_{\mathbf{x}}(\boldsymbol{\lambda})$ are Lipschitz continuous with Lipschitz constant $M$

$$
\left\|\nabla g_{\mathbf{x}}(\boldsymbol{\lambda})-\nabla g_{\mathbf{x}}(\boldsymbol{\mu})\right\| \leq M\|\boldsymbol{\lambda}-\boldsymbol{\mu}\|
$$

(A3) The 2-norm of the dual gradients $\nabla g_{\mathbf{x}}(\boldsymbol{\lambda})$ are uniformly bounded for all $\boldsymbol{\lambda}$ and all $\mathrm{x}$,

$$
\left\|\nabla g_{\mathbf{x}}(\boldsymbol{\lambda})\right\| \leq G_{\max }
$$

(A4) The 1-norm of the optimal Lagrange multipliers $\lambda_{\mathbf{x}}^{*}$ are uniformly bounded for all $\mathbf{x}$

$$
\left\|\boldsymbol{\lambda}_{\mathbf{x}}^{*}\right\|_{1} \leq \lambda_{\max }
$$

These assumptions are mild, technical, and commonly required in the analysis of gradient descent algorithms. Since the iteration in (17) implements dual gradient descent, the main result in this section describes the distance between the current Lagrange multiplier $\boldsymbol{\lambda}\left(t_{n}\right)$ and the current optimal Lagrange multiplier $\boldsymbol{\lambda}_{\mathbf{x}\left(t_{n}\right)}^{*}$ as stated next ${ }^{1}$.

Theorem 1 Let $\mathrm{x}\left(t_{n}\right)$ denote the configuration at iteration $n, \boldsymbol{\lambda}_{\mathbf{x}\left(t_{n}\right)}^{*}$ the corresponding optimal dual variable and $\boldsymbol{\lambda}\left(t_{n}\right)$ the dual iterate obtained through iterative application of (17). Assume the step size in (17) is bounded as $\epsilon \leq 1 / M$ and that the difference between reliabilities at subsequent configurations is absolutely bounded by $\delta>0$, i.e.,

$$
\left|R_{i j}\left(\mathbf{x}\left(t_{n+1}\right)\right)-R_{i j}\left(\mathbf{x}\left(t_{n}\right)\right)\right| \leq \delta .
$$

If assumptions (A1)-(A4) hold, the distance between the dual iterate $\boldsymbol{\lambda}\left(t_{n}\right)$ and the optimal multiplier $\boldsymbol{\lambda}_{\mathbf{x}\left(t_{n}\right)}^{*}$ satisfies

$$
\left\|\boldsymbol{\lambda}\left(t_{n}\right)-\boldsymbol{\lambda}_{\mathbf{x}\left(t_{n}\right)}^{*}\right\| \leq \beta^{n}\left\|\boldsymbol{\lambda}\left(t_{0}\right)-\boldsymbol{\lambda}_{\mathbf{x}\left(t_{0}\right)}^{*}\right\|+\frac{2 \lambda_{\max } J}{m(1-\beta)} \delta,
$$

where the constant $\beta$ is defined as $\beta:=\sqrt{1 /(1+m \epsilon)}$.

Theorem 1 dictates that dual iterates $\boldsymbol{\lambda}\left(t_{n}\right)$ converge linearly to a neighborhood of the optimal multiplier $\boldsymbol{\lambda}_{\mathbf{x}\left(t_{n}\right)}^{*}$. The volume of this neighborhood is determined by problemspecific constants and can be made arbitrarily small by reducing $\delta$, which can be controlled by modulating the velocity of the robots. Results of interest for the primal variables follow as corollaries of the result in Theorem 1. The feasibility gap of $\mathbf{t}\left(t_{n}\right), \mathbf{r}\left(t_{n}\right)$ is bounded in Corollary 1 and a similar bound for the optimality gap is presented in Corollary 2.

Corollary 1 With the hypotheses and definitions of Theorem 1 , the norm of the constraint violation can be bounded as

$$
\begin{aligned}
\left\|P\left(\mathbf{r}\left(t_{n}\right)-\mathbf{A}_{\mathbf{x}\left(t_{n}\right)} \mathbf{t}\left(t_{n}\right)\right)\right\| \leq & M \beta^{n}\left\|\boldsymbol{\lambda}\left(t_{0}\right)-\boldsymbol{\lambda}_{\mathbf{x}\left(t_{0}\right)}^{*}\right\| \\
& +\frac{2 M \lambda_{\max } J}{m(1-\beta)} \delta
\end{aligned}
$$

Corollary 2 Let $f_{0}\left(t_{n}\right):=f_{0}\left(\mathbf{t}\left(t_{n}\right), \mathbf{r}\left(t_{n}\right)\right)$ denote the primal objective corresponding to the communication variables at time $t_{n}$. With the same hypotheses and definitions of Theorem 1, the optimality gap can be bounded as

$$
\begin{aligned}
P_{\mathbf{x}\left(t_{n}\right)}-f_{0}\left(t_{n}\right) \leq & \left(G_{\max }+\lambda_{\max } M\right) \beta^{n}\left\|\boldsymbol{\lambda}\left(t_{0}\right)-\boldsymbol{\lambda}_{\mathbf{x}\left(t_{0}\right)}^{*}\right\| \\
+ & +\frac{2\left(G_{\max }+\lambda_{\max } M\right) \lambda_{\max } J}{m(1-\beta)} \delta
\end{aligned}
$$

\footnotetext{
${ }^{1}$ Proofs are available in the journal version of this paper.
} 


\section{A. Network integrity}

Theorem 1 and Corollaries 1 and 2 guarantee that the communication variables are close to optimality and feasibility at sampling times $t_{n}$. This proximity depends on the disturbance $\left|R_{i j}\left(\mathbf{x}\left(t_{n+1}\right)\right)-R_{i j}\left(\mathbf{x}\left(t_{n}\right)\right)\right|=\delta>0$ introduced in (2) due to robot mobility between consecutive communication updates at times $t_{n}$ and $t_{n+1}$. To extend these results to all time $t \geq t_{0}$ let

$$
\mathcal{S} \triangleq\left\{\mathbf{x}(t) \in \mathbb{R}^{J d} \mid \tilde{\beta}_{i}\left(t ; \sigma_{i}(t)\right)>0, \forall i=1, \ldots, J\right\},
$$

where $\tilde{\beta}_{i}\left(t ; \sigma_{i}(t)\right)$ is defined as in (15), denote the set of robot configurations $\mathbf{x}(t)$ that ensure communication rates $r_{i}(t)$ that exceed desired basal rates $r_{i 0}$ with some error $e_{i}>0$, i.e., $r_{i}(t)>r_{i 0}-e_{i}$, as required in Problem 1. Moreover, assume that the channel reliabilities $R\left(\mathbf{x}_{i}(t), \mathbf{x}_{j}(t)\right)$ are purely a function of the inter-robot distances and let $M_{R}=$ $\max _{i, j}\left\{\left|\frac{d R_{i j}(\mathbf{x})}{d\left\|\mathbf{x}_{i j}\right\|_{2}}\right|\right\}$, where $\mathbf{x}_{i j}=\mathbf{x}_{i}-\mathbf{x}_{j}$, denote their absolute maximum slope. Let also $M_{\phi}=\max _{i}\left\{\left\|\nabla_{\mathbf{x}_{i}} \phi_{i}\right\|_{2}\right\}$ denote the maximum robot speed. Then, we have the result:

Theorem 2 If $\mathbf{x}\left(t_{0}\right) \in \mathcal{S}$, then $\mathbf{x}(t) \in \mathcal{S}$ for all time $t \geq t_{0}$. Moreover, the approximation error satisfies

$$
e_{i} \leq 2 k \Delta t M_{R} M_{\phi} \frac{2 M \lambda_{\max } J}{m(1-\beta)},
$$

for all time $t \geq t_{0}$, where $\Delta t=\max _{n}\left\{t_{n+1}-t_{n}\right\}$.

Clearly, the error upper bound in Theorem 2 can be made arbitrarily small by appropriately tuning the robot speed gain $k>0$ and the frequency of communication $t_{n+1}-t_{n}$. The smaller this bound is, the closer to optimality will every primal-dual iteration (9)-(10) be. Since the frequency of communication is typically subject to practical constraints, this implies that the speed gain $k>0$ might need to be sufficiently small to realize the desired bounds.

\section{INTEGRATION WITH ROBOT TASKS}

In this section we illustrate our approach in a nontrivial scenario where communication integrity of the robot network needs to be preserved. For this, we employ channel reliabilities $R_{i j}=R\left(\mathbf{x}_{i}, \mathbf{x}_{j}\right)$ that are twice differentiable and monotonically decreasing functions of $\mathbf{x}_{i j} \triangleq \mathbf{x}_{i}-\mathbf{x}_{j}$ between 1 and 0 . We consider a scenario where reliable communication is necessary between $K=2$ access points (APs) and two service point (SP) in $\mathbb{R}^{2}$. This task is implemented by a team of $J=15$ robots, two of which are leaders responsible for serving the SPs. The task potentials $\gamma_{i}$ are identically set to 1 for all robots except for the leader robots for which $\gamma_{i} \triangleq \frac{1}{2}\left\|\mathbf{x}_{i}-\mathbf{x}_{i}^{d}\right\|_{2}^{2}$, where $\mathbf{x}_{i}^{d}$ denotes the location of each service point. Finally, the minimum rates $r_{i, 0}$ are identically zero for all robots except for the leaders for which $r_{i, 0}=0.95$. These values are consistent with the classification of robots into relays and leaders. Leaders collect measurements and generate data, while relays forward this information to the APs. Results are shown in Fig. 2. We observe that the leader robots (labeled as 3 and 4) move towards the SPs and force the network to stretch in order to achieve reliable communication between the SPs and APs.

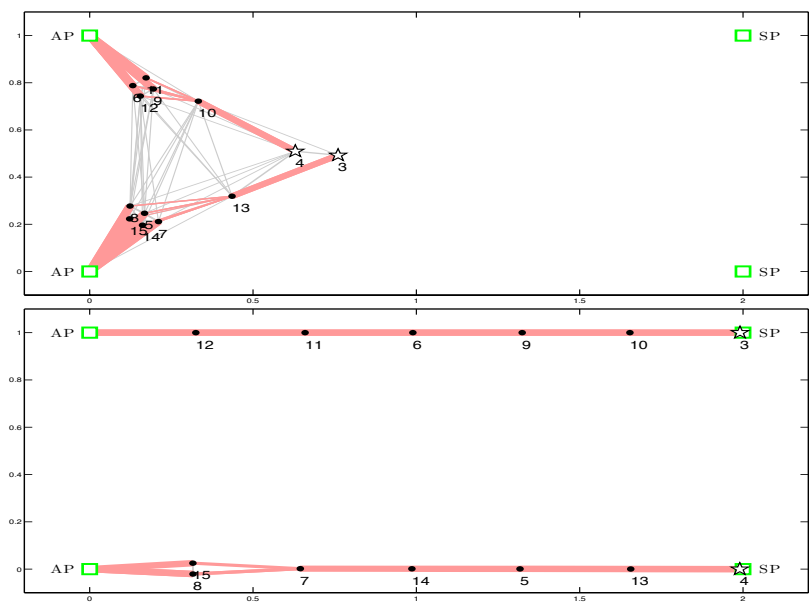

Fig. 2. Mobile robot network consisting of 15 robots (dots), 2 service points (SP) and 2 access points (AP). The grey lines represent channel reliabilities $R\left(\mathbf{x}_{i}, \mathbf{x}_{j}\right)$ and the red lines represent routing of information $T_{i j} R\left(\mathbf{x}_{i}, \mathbf{x}_{j}\right)$, between pairs of robots. The thickness of each line represents the magnitude of these quantities. The network is randomly initialized and involves 2 leaders (stars), labeled 3 and 4, that move towards the upper and lower service points, respectively.

\section{CONCLUSIONS}

In this paper we considered the problem of ensuring communication integrity in networks of mobile robots. Our approach relied on introducing weights on the communication links to capture channel reliabilities, which then allowed to model routing by means of optimization problems that accept link reliabilities as inputs. The key idea proposed in this work was the joint control of mobility and communications in a hybrid scheme with the discrete-time routing variables being the switching signal in the continuous-time motion controllers. We provided communication guarantees within a bounded error of optimality.

\section{REFERENCES}

[1] R. Olfati-Saber, J. A. Fax, and R. M. Murray, "Consensus and cooperation in networked multi-agent systems," Proc. of the IEEE, vol. 95, no. 1, pp. 215-233, January 2007.

[2] K. Pahlavan and A. H. Levesque, Wireless Information Networks. New York, NY: John Willey \& Sons, 1995.

[3] M. Ji and M. Egerstedt, "Coordination control of multi-agent systems while preserving connectedness," IEEE Transactions on Robotics, vol. 23, no. 4, pp. 693-703, August 2007.

[4] M. M. Zavlanos and G. J. Pappas, "Potential fields for maintaining connectivity of mobile networks," IEEE Transactions on Robotics, vol. 23, no. 4, pp. 812-816, August 2007.

[5] — , "Distributed connectivity control of mobile networks," IEEE Transactions on Robotics, vol. 24, no. 6, pp. 1416-1428, December 2008.

[6] H. Lundgren, E. Nordstrom, and C. Tschudin, "The gray zone problem in ieee 802.11 b based ad hoc networks," ACM SIGMOBILE Mobile Computing and Communications Review, vol. 6, no. 3, pp. 104-105, July 2002.

[7] D. DeCouto, D. Aguayo, J. Bicket, and R. Morris, "A high-throughput path metric for multihop wireless routing," in Proc. ofInternational ACM Conference on Mobile Computing and Networking, San Diego, CA, September 2006, pp. 134-146.

[8] A. Ribeiro, N. D. Sidiropoulos, and G. B. Giannakis, "Optimal distributed stochastic routing algorithms for wireless multihop networks," IEEE Transactions on Wireless Communications, vol. 7, no. 11, pp. 4261-4272, November 2008.

[9] N. Z. Shor, Minimization Methods for Non-Differentiable Functions. Berlin, Heilderberg, Germany: Springer-Verlag, 1985. 